\title{
Atypical hand, foot, and mouth disease in an adult patient: a case report and literature review
}

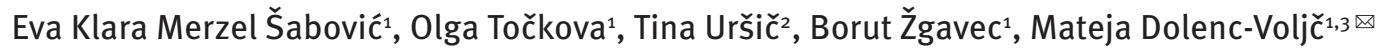

\begin{abstract}
Hand, foot, and mouth disease is a highly contagious disease that predominantly affects children under the age of five. The illness is much less common in adults and often presents with atypical skin lesions. Here we describe the case of a 19-year-old healthy, immunocompetent male patient with atypical clinical presentation, with a macular rash on the scalp, palms, soles, and shins, and with minimal lesions in the oral cavity. The diagnosis of hand, foot, and mouth disease was confirmed by isolation of coxsackievirus A6 from a vesicle smear obtained on the right sole. The skin lesions resolved spontaneously in 1 week. This case emphasizes the underestimated fact that hand, foot, and mouth disease can also occur in adults, in either typical or frequently atypical form, and it highlights the role of coxsackievirus A6. Together with the case presentation, a review of the literature describing the disease in adults is presented.
\end{abstract}

Keywords: hand, foot, and mouth disease, coxsackievirus A6, enterovirus

Received: 8 February 2019| Returned for modification: 1 April 2019| Accepted: 10 April 2019

\section{Introduction}

Hand, foot, and mouth disease (HFMD) is a childhood disease primarily affecting children under age 10, most commonly younger than five $(1,2)$. The disease is epidemic in Asia, especially in China, with an incidence rate of around 500,000 to 1,000,000 cases per year (3). Classic HFMD, with typical oral enanthem, followed by maculopapular exanthema with vesicles on the palms and soles, is most commonly caused by enterovirus 71 (EV71) and coxsackievirus A16 (CVA16) (4). Other coxsackieviruses have also been associated with HFMD: CVA5, CVA6, CVA9, B2, and B5 (5, 6). Recently, new serotypes of enteroviruses that have caused outbreaks of HFMD worldwide have been reported, especially CVA6 and CVA10 (7-9). The clinical presentation of a CVA6-related HFMD differs from classic HFMD; the distribution can be more widespread, with purpuric lesions (10). Therefore, due to both atypical clinical presentation of CVA6-related HFMD and perceiving HFMD as a childhood disease, there can be a delay in establishing the diagnosis in adult cases and consequent increased risk of spread of the disease. We present the case of an adult male with atypical presentation of HFMD, as well as a review of literature addressing HFMD in adults and particularly in CVA6-related HFMD.

\section{Case report}

A 19-year-old healthy male patient was referred to our department with a suspicion of erythema multiforme and a fever up to $39.5{ }^{\circ} \mathrm{C}$. Before the onset of the disease, the patient attended an international sports competition, at which young children were also competing, but he did not report close contact with an evidently ill child or adult. The fever started 1 day before admission. He took four pills of paracetamol $500 \mathrm{mg}$ but denied taking any other medication or over-the-counter remedies. The next day, pruritic skin lesions appeared, first around the mouth and on the chin, nose, and forehead, and a few on the cheeks and later concomitantly on the scalp, palms, and soles, and less intensively on the shins and wrists. Cutaneous lesions presented in different stages-from erythematous macules and solitary papules, to rare vesicles, erosions, and crusts - and they varied in size and color. Vesicles were mostly found around the mouth (Fig. 1), a few were on the palms, and one was on the right shin above the Achilles tendon (Fig. 2). On the palms and soles, on the dorsum of the hands and feet, and on the shins, purpuric macules were the predominant lesions (Figs. 2-4). Interestingly, diffuse macular and scaly lesions were also present on the scalp. Two erosions were located in the oral cavity, which did not cause any oral discomfort. No history or clinical signs of immunodeficiency or atopy were demonstrated.

Laboratory findings revealed elevated C-reactive protein in serum $(55 \mathrm{mg} / \mathrm{l})$, whereas all other laboratory findings, including white blood cell count, hemoglobin, liver tests, renal function, myoglobin, creatinine kinase, and urine analysis, were unremarkable, as was chest X-ray. A smear of the perioral crusts for pathogenic bacteria was negative. Serologic assay by complement fixation and PCR for parvovirus, and PCR for varicella zoster virus, herpes-

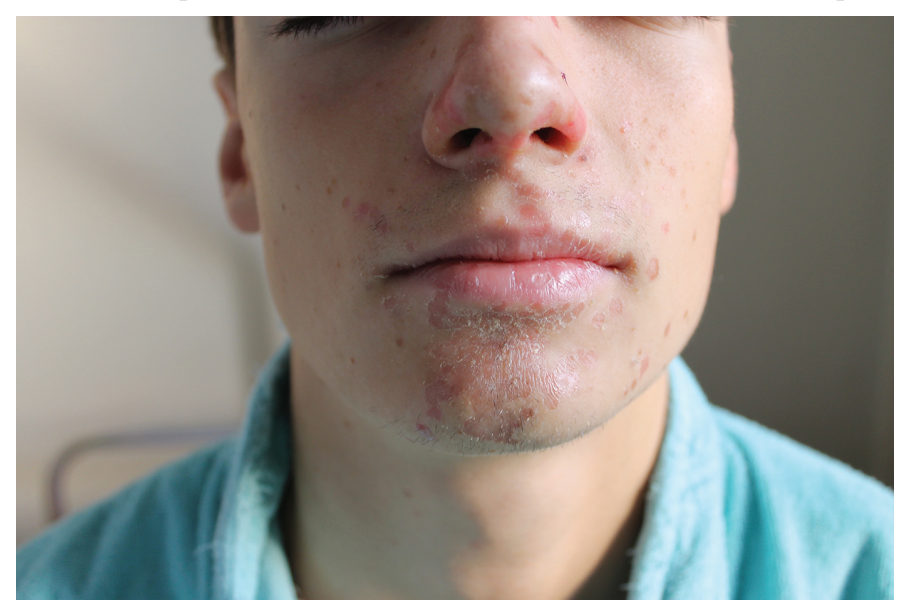

Figure 1 | Erythematous macules, rare vesicles, erosions, and crusts around the mouth. 


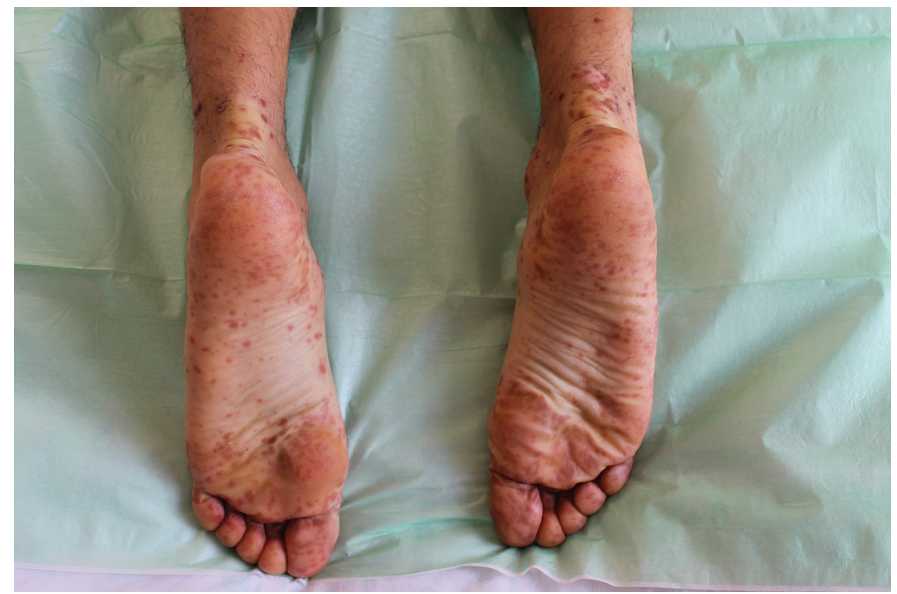

Figure 2 | Purpuric macules on the soles, a vesicle above the right Achilles tendon.

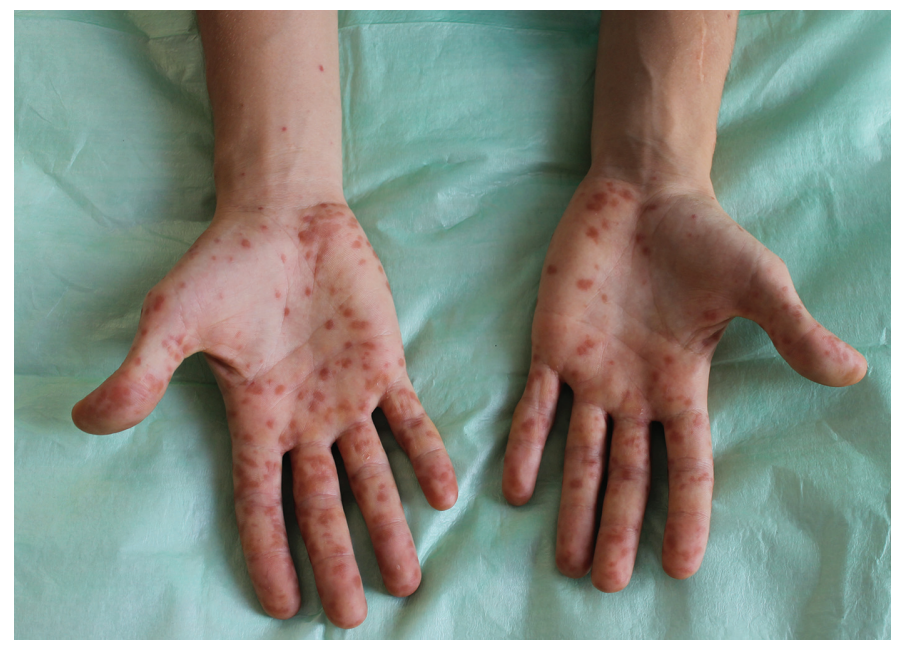

Figure 3 | Purpuric macules on the palms.

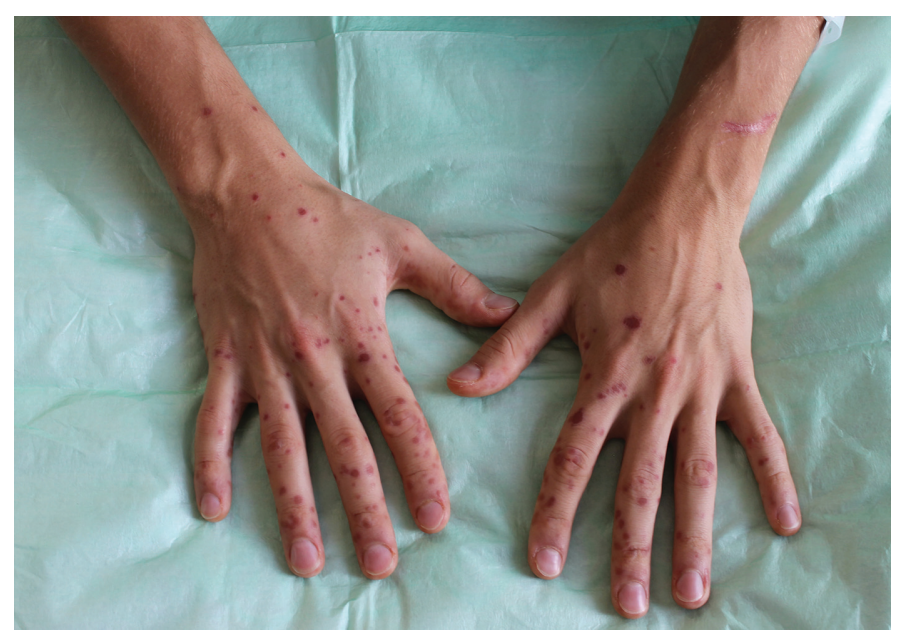

Figure 4 | Purpuric macules on the dorsum of the hands.

simplex virus 1, herpes-simplex virus 2, and Mycoplasma (buccal swab) were all negative. However, PCR from a vesicle smear, obtained from a vesicle on the right sole, revealed enterovirus, and further analysis by sequencing revealed CVA6.

Due to the atypical clinical presentation, a skin biopsy was performed. Histopathological examination revealed a slight epidermal acanthosis with scattered necrotic keratinocytes, covered with remnants of confluent necrosis and basket-weave stratum corneum. In the dermis, superficial perivascular, mostly mononuclear infiltrate with evident extravasated erythrocytes and focal discrete interface dermatitis with some basal vacuolar changes was observed.

Based on isolation of CVA6 and the clinical presentation, a diagnosis of HFMD was made. The patient was treated topically with fusidic acid cream on the face and with a combined cream of betamethasone and gentamicin on the palms. Due to itchiness he also received peroral antihistamines. After 1 day, his temperature normalized spontaneously and the skin changes regressed over the next 4 days. He was discharged after 4 days with residual macules and in good general condition.

\section{Discussion}

Classic HFMD is a common childhood illness, whereas it is sporadic in adults. The disease is highly contagious among children, but much less so in adults (11). It is more prevalent in immunocompromised patients. Atopy is also considered a risk factor for disease occurrence in adults. In immunocompetent adults, infection with a more virulent strain of an enterovirus should be suspected (11). CVA6 seems to be the most aggressive, having the capacity to produce a worse clinical course of the disease and atypical clinical manifestations $(10,12)$. Before 2008, studies indicated that CVA6 mainly caused herpangina. It was first identified in HFMD in 2008 in Finland, where it caused a major HFMD outbreak $(7,13)$. Since then, CVA6 has become the major pathogen causing outbreaks in Europe, Asia, and the United States (8, 9, 12, 14-16). CVA6-associated HFMD has a high frequency of onset in winter and high morbidity in adults.

HFMD is transmitted via a fecal-oral route, through oral-pharyngeal secretions, or by direct skin contact (17). The disease usually occurs in spring and summer. The incubation period ranges from 2 to 7 days. Prodromal symptoms are usually absent or include mild fever, catarrhal symptoms, abdominal pain, emesis, and diarrhea (18). The typical clinical presentation is painful oral enanthem, followed by skin lesions on distal extremities, especially on the palms and soles, of varying morphology (macules, papules, vesicles, and crusts), size, and color (5). The diagnosis is usually made clinically by the characteristic distribution and type of skin lesions (19). Differential diagnosis of oral lesions includes aphthous ulcers and primary herpes simplex gingivostomatitis, whereas in the case of maculopapular or vesicular rashes, chickenpox, id reaction, erythema exudativum multiforme, and eczema herpeticum should be considered $(19,20)$. The treatment is supportive (19). In most cases, the disease is self-limiting and has no sequelae (4). Complications are rare, although encephalitis, myoclonic seizure, pulmonary edema, or even death due to cardiopulmonary failure may develop (19).

Our patient had an unusual clinical presentation. The following atypical features were found: i) no typical history or risk factors, ii) involvement of atypical regions: the dorsum of the hands and feet, scalp, and perioral region, iii) only a few lesions in the oral cavity, and iv) a different pattern of skin lesions: predominantly purpuric macules, whereas typical vesicles and erosions were rare. Due to the listed features, the diagnosis in our patient was not simply straightforward at the beginning. Similar clinical presentations, with perioral involvement, skin lesions on the dorsum of the hands and feet, and purpura-like palmar-plantar macules, have also been reported in other patients with CVA6-related $\operatorname{HFMD}(6,21)$. The unique feature of our case was the presence of macular and scaly lesions also on the scalp.

In the literature, numerous cases of HFMD have been reported in children, whereas adult cases have been much less frequently 
reported. The clinical presentation and the course of the disease in adults, particularly in those affected by CVA6, can be different than the course in children. In CVA6-related HFMD, the patient first experiences prodromal symptoms, which can range from mild to severe, including high fever $\left(>38.5^{\circ} \mathrm{C}\right)$ and gastrointestinal and catarrhal symptoms (21). Prodromal symptoms are then followed by oral lesions or, more commonly, pruritic perioral vesicles or erythematous or purpura-like macular lesions on the palms and soles, which can mimic those of secondary syphilis (22). A finding specific for CVA6 is the involvement of the dorsum of the hands and feet, in contrast to classic HFMD (10, 22). After several weeks, patients can experience desquamation of the palms and soles and/or onychomadesis (9). A study from Taiwan revealed that $51 \%$ of patients with CVA6-related HFMD experienced desquamation of the palms and soles after several weeks, and $37 \%$ of them developed onychomadesis (9). There is also a higher incidence rate for complications after HFMD caused by CVA6; among the most threatening is encephalitis (7). EV71 and CVA1o have also been associated with neurological complications (encephalitis) and death $(4,23)$. Differences in the clinical presentation of classic and CVA6-related HFMD are presented in Table 1.

Due to the different clinical presentations of classic HFMD and CVA6-related HFMD, adults with HFMD are commonly erroneously diagnosed. In one study from Denmark, out of 23 patients, only one patient was suggested to have HFMD in the referral notes (21). Other diagnoses were erythema multiforme, syphilis, Stevens-Johnson syndrome, eczema herpeticum, dermatophytid, psoriasis, vasculitis, impetigo, and atopic dermatitis (21). HFMD caused by CVA6 is therefore called a "diagnostic mimicker" in the literature because it resembles many other skin diseases.

The diagnostic workup in adults is similar as in children, but confirmation of the diagnosis can be delayed in adults and the isolation of causative enteroviruses is more often required. Confirmation of diagnosis is performed by isolation of the virus from vesicle fluid, oropharyngeal swabs, or stool samples, or by identifying virus-neutralizing antibodies in the patient's serum (19). In addition, virus subtyping can be performed.

When the biopsy is obtained, typical histopathological findings for HFMD are reticular and ballooning degeneration of the epidermis (11). A biopsy specimen was taken from an already regressive, previously vesicular lesion in our patient. Histopatho- logical findings were not entirely specific, but could be consistent with an older HFMD lesion as well as erythema multiforme in our patient. However, according to some authors, large numbers of neutrophils in parakeratosis in the viable epidermis or neutrophils forming intraepidermal collections, as well as more numerous extravasated erythrocytes in the epidermis and necrosis more emphasized in the upper part of the epidermis, are features more common in HFMD (24), which was also present in our case to some extent. The main histopathological difference between classic and CVA6-related HFMD is extensive necrosis of the epidermis, present in CVA6-related HFMD (21), as also observed in our patient.

In relation to epidemiology, HFMD has been a massive public health concern in India and Southeast Asia due to the high incidence of cases and common epidemic outbreaks (3). Numerous studies have been performed aimed at identifying predictive factors that could anticipate possible outbreaks in Asia (25-28). Overall, meteorological factors such as temperature and humidity have been identified as possible predictive factors for the occurrence of outbreaks (29).

Due to the epidemic prevalence of HFMD in Asia, vaccines for EV71 and CVA16, but not for CVA6, have recently been developed and tested in humans $(4,19,30,31)$. However, due to a change in the subtype of enteroviruses that have recently caused major HFMD outbreaks, particularly CVA6, control of the disease has been challenging (4). Preventive strategies have also been explored, such as closure of schools and childcare centers, handwashing routines, and airing bedding $(28,32)$. It has been revealed that only regular handwashing has a significant protective value for prevention of HFMD (33).

In summary, we present an adult, immunocompetent male patient with an unusual clinical manifestation of HFMD caused by CVA6. In contrast to the typical clinical presentation in children, the clinical presentation in adults, and especially in CVA6-related HFMD, is often atypical and may mimic other acute skin diseases. When examining an adult patient presenting with fever and an abrupt maculopapular rash affecting the palms and soles or dorsal parts of the hands and feet, HFMD should therefore also be considered in the differential diagnosis. In view of the contagious nature of HFMD, early diagnosis and prevention of its spread are necessary.

Table 1 | Comparison of clinical characteristics between classic and CVA6-related HFMD.

\begin{tabular}{lcc}
\hline Clinical characteristics & Classic HFMD & CVA6-related HFMD \\
\hline Age of patients & Children $<7$ years & Predominantly adults \\
Peak incidence & Summer and spring & Winter \\
Skin lesions & Maculopapular lesions with vesicles & Purpuric lesions \\
Skin lesion distribution & Oral cavity, palms and soles (palmar site) & More widespread (perioral area, dorsum of hands and feet, trunk, extremities) \\
Complications & Rare & More common (meningitis, encephalitis) \\
Prodromal symptoms & Rare; if present, mild & Often higher fever $\left(>38.5^{\circ} \mathrm{C}\right)$ \\
\hline
\end{tabular}

Prodromal symptoms ; CVA16 = coxsackievirus A16.

\section{References}

1. Li LJ. Review of hand, foot and mouth disease. Front Med China. 2010;4:139-46.

2. Chen KT, Chang HL, Wang ST, Cheng YT, Yang JY. Epidemiologic features of handfoot-mouth disease and herpangina caused by enterovirus 71 in Taiwan, 19982005. Pediatrics. 2007;120:e244-52.

3. Wang JF, Guo YS, Christakos G, Yang WZ, Liao YL, Li ZJ, et al. Hand, foot and mouth disease: spatiotemporal transmission and climate. Int J Health Geogr. 2011;5:10-25.

4. Aswathyraj S, Arunkumar G, Alidjinou EK, Hober D. Hand, foot and mouth disease (HFMD): emerging epidemiology and the need for a vaccine strategy. Med Microbiol Immunol. 2016;205:397-407.
5. Yang F, Zhang T, Hu Y, Wang X, Du J, Li Y, et al. Survey of enterovirus infections from hand, foot and mouth disease outbreak in China, 2009. Virol J. 2011;8:1-4. 6. Lott JP, Liu K, Landry ML, Nix WA, Oberste MS, Bolognia J, et al. Atypical handfoot-mouth disease associated with coxsackievirus A6 infection. J Am Acad Dermatol. 2013;69:736-41.

7. Blomqvist S, Klemola P, Kaijalainen S, Paananen A, Simonen ML, Vuorinen T, et al. Co-circulation of coxsackieviruses $A 6$ and $A 10$ in hand, foot and mouth disease outbreak in Finland. J Clin Virol. 2010;48:49-54. 
8. Mirand A, Henquell C, Archimbaud C, Ughetto S, Antona D, Bailly JL, et al. Outbreak of hand, foot and mouth disease / herpangina associated with coxsackievirus $A 6$ and $A 10$ infections in 2010, France: a large citywide, prospective observational study. Clin Microbiol Infect. 2012;18:e110-8.

9. Wei SH, Huang YP, Liu MC, Tsou TP, Lin HC, Lin TL, et al. An outbreak of coxsackievirus A6 hand, foot, and mouth disease associated with onychomadesis in Taiwan, 2010. BMC Infect Dis. 2011;11:346.

10. Ramirez-Fort MK, Downing C, Doan HQ, Benoist F, Oberste MS, Khan F, et al. Coxsackievirus A6 associated hand, foot and mouth disease in adults: clinical presentation and review of the literature. J Clin Virol. 2014;60:381-6.

11. Shin JU, Oh SH, Lee JH. A case of hand-foot-mouth disease in an immunocompetent adult. Ann Dermatol. 2010;22:216-8.

12. Fujimoto T, lizuka S, Enomoto M, Abe K, Yamashita K, Hanaoka N, et al. Hand, foot, and mouth disease caused by coxsackievirus A6, Japan. 2011. Emerg Infect Dis. 2012;18:337-9.

13. Osterback R, Vuorinen T, Linna M, Susi P, Hyypia T, Waris M. Coxsackievirus A6 and hand, foot, and mouth disease, Finland. Emerg Infect Dis. 2009;15:1285-8.

14. Lu QB, Zhang XA, Wo Y, Xu HM, Li XJ, Wang Xj, et al. Circulation of coxsackievirus $A 10$ and $A 6$ in hand-foot-mouth disease in China, 2009-2011. PloS One. 2012; 7:e52073.

15. Buttery VW, Kenyon C, Grunewald S, Oberste MS, Nix WA. Atypical presentations of hand, foot, and mouth disease caused by coxsackievirus A6 - Minnesota, 2014. MMWR Morb Mortal Wkly Rep. 2015;64:805.

16. Bian L, Wang Y, Yao X, Mao Q, Xu M, Liang Z. Coxsackievirus A6: a new emerging pathogen causing hand, foot and mouth disease outbreaks worldwide. Expert Rev Anti Infect Ther. 2015:13:1061-71.

17. Park SK, Park B, Ki M, Kim H, Lee K, Jung C, et al. Transmission of seasonal outbreak of childhood enteroviral aseptic meningitis and hand-foot-mouth disease. J Korean Med Sci. 2010;25:677-83.

18. Liu MY, Liu W, Luo J, Liu Y, Zhu Y, Berman H, et al. Characterization of an outbreak of hand, foot, and mouth disease in Nanchang, China in 2010. PloS One. 2011; 6:e25287.

19. Omana-Cepeda C, Martinez-Valverde A, del Mar Sabater-Recolons M, Jane-Salas E, Mari-Roig A, Lopez-Lopez J. A literature review and case report of hand, foot and mouth disease in an immunocompetent adult. BMC Res Notes. 2016;9:165.

20. Muppa R, Bhupatiraju P, Duddu M, Dandempally A. Hand, foot and mouth disease. J Indian Soc Pedod Prev Dent. 2011;29:165-7.

21. Horsten HH, Kemp M, Fischer TK, Lindahl KH, Bygum A. Atypical hand, foot, and mouth disease caused by coxsackievirus A6 in Denmark: a diagnostic mimicker. Acta Derm Venereol. 2018;98:350-4.
22. Feder HM Jr, Bennett N, Modlin JF. Atypical hand, foot, and mouth disease: a vesiculobullous eruption caused by Coxsackie virus A6. Lancet Infect Dis. 2014;14: 83-6.

23. Chong CY, Chan KP, Shah VA, Ng WY, Lau G, Teo TE, et al. Hand, foot and mouth disease in Singapore: a comparison of fatal and non-fatal cases. Acta Pediatrica. 2003;92:1163-9.

24. Böer-Auer A, Metze D. Histopathology of hand-foot-mouth disease in adults and criteria for differentiation from erythema multiforme. Am J Dermatopathol. 2019;41:273-80.

25. Zhang X, Xu C, Xiao G. Space-time heterogeneity of hand, foot and mouth disease in children and its potential driving factors in Henan, China. BMC Infect Dis. 2018;18:638.

26. Coates SJ, Davis MDP, Andersen LK. Temperature and humidity affect the incidence of hand, foot, and mouth disease: a systematic review of the literature-a report from the International Society of Dermatology Climate Change Committee. Int J Dermatol. 2019;58:388-99.

27. Qi H, Chen Y, Xu D, Su H, Zhan L, Xu Z, et al. Impact of meteorological factors on the incidence of childhood hand, foot, and mouth disease (HFMD) analyzed by DLNMs-based time series approach. Infect Dis Poverty. 2018;7:7.

28. Koh WM, Bogich T, Siegel K, Jin J, Chong EY, Tan CY, et al. The epidemiology of hand, foot and mouth disease in Asia-a systematic review and analysis. Pediatr Infect Dis J. 2016;35:e285-300.

29. Zou XN, Zhang XZ, Wang B, Qiu YT. Etiologic and epidemiologic analysis of hand, foot, and mouth disease in Guangzhou city: a review of 4,753 cases. Braz J Infect Dis. $2012 ; 16: 457-65$.

30. Zhu F, Xu W, Xia J, Liang Z, Liu Y, Zhang X, et al. Efficacy, safety, and immunogenicity of an enterovirus 71 vaccine in China. N Engl J Med. 2014;370:818-28.

31. Zhu FC, Meng FY, Li JX, Li XL, Mao QY, Tao H, et al. Efficacy, safety, and immunology of an inactivated alum-adjuvant enterovirus 71 vaccine in children in China: a multicentre, randomised, double-blind, placebo-controlled, phase 3 trial. Lancet. 2013;381:2024-32.

32. Chen Y, Badaruddin H, Lee VJ, Cutter J, Cook AR. The effect of school closure on hand, foot, and mouth disease transmission in Singapore: a modeling approach. Am J Trop Med Hyg. 2018;99:1625-32.

33. Zhang D, Li Z, Zhang W, Guo P, Ma Z, Chen Q, et al. Hand-washing: the main strategy for avoiding hand, foot and mouth disease. Int J Environ Res Public Health. 2016;13:610. 